\title{
Double-Stranded Break Can Be Repaired by Single-Stranded Oligonucleotides via the ATM/ATR Pathway in Mammalian Cells
}

\author{
ZAI WANG, ${ }^{1}$ ZHONG-JUN ZHOU,${ }^{1}$ DE-PEI LIU, ${ }^{2}$ and JIAN-DONG HUANG ${ }^{1}$
}

\begin{abstract}
Single-stranded oligonucleotide (SSO)-mediated gene modification is a newly developed tool for sitespecific gene repair in mammalian cells; however, the corrected cells always show G2/M arrest and cannot divide to form colonies. This phenomenon and the unclear mechanism seriously challenge the future application of this technique. In this study, we developed an efficient SSO-mediated DNA repair system based on double-stranded break (DSB) induction. We generated a mutant EGFP gene with insertions of $24 \mathrm{bp}$ to $1.6 \mathrm{~kb}$ in length as a reporter integrated in mammalian cell lines. SSOs were successfully used to delete the insertion fragments upon DSB induction at a site near the insertion. We demonstrated that this process is dependent on the ATM/ATR pathway. Importantly, repaired cell clones were viable. Effects of deletion length, SSO length, strand bias, and SSO modification on gene repair frequency were also investigated.
\end{abstract}

\section{INTRODUCTION}

$S^{\prime}$ into mammalian cells (Igoucheva et al. 2001; Liu et al. 2001; Lu et al., 2003). Up to now, the repair process has not been fully understood. Our previous study has shown that SSO may anneal to its homologous target during DNA replication (Wu et al., 2005), and the recombination used pathways other than the ATM/ATR pathway (Wang et al. 2006). However, the corrected cells always show G2/M arrest and cannot divide to form colonies (Olsen et al., 2005a). This serious problem and the unclear reason are hurdles to the future application of this technique.

In yeast, SSOs are able to mediate long fragment deletion, and very high efficiencies have been achieved upon site-specific double-stranded break (DSB) induction (Storici et al. 2003). Similarly, in mammalian cells, a recent report showed that SSOs can modify chromosomal gene in the presence of a DSB and generate viable clones (Radecke et al., 2006). However, which recombination pathway the SSOs utilize is not clear. Furthermore, for future applications of this method, there are several parameters that need to be optimized to achieve the highest efficiency.

For this purpose, we used a series of mutant EGFP genes harboring insertions of $24 \mathrm{bp}$ to $1.6 \mathrm{~kb}$ in length as a reporter system. We showed SSOs can successfully delete the inserted fragment upon DSB induction, and generate viable cell clones after chromosomal repair. We demonstrated that this process depends on the ATM/ ATR pathway. Effects of parameters related to recombination efficiency were also tested.

\footnotetext{
${ }^{1}$ Department of Biochemistry, The University of Hong Kong, Hong Kong SAR, People's Republic of China.

${ }^{2}$ National Laboratory of Medical Molecular Biology, Institute of Basic Medical Sciences, Chinese Academy of Medical Sciences (CAMS) and Peking Union Medical College (PUMC), Beijing 100005, People's Republic of China.
} 


\section{MATERIALS AND METHODS}

\section{Plasmids}

To generate pcSceGFP-24, the first fragment of SceGFP sequence was amplified from pDRGFP (Pierce et al., 1999) using primers: 5'-CGGGATCCGCCACCATGGTGAGCAA-3' (forward), and 5'-GCGAATTCATTACCCTGTTATCCCTAGC-3' (reverse), and then digested with BamHI and EcoRI. Underlined nucleotides are the recognition site of $I-S c e I$, which also introduces two in-frame stop codons. The second fragment of SceGFP was amplified from pEGFP-C1 (Clontech, Palo Alto, CA) using primers: 5'-GCGAATTCGAGGGCGAGGGCGATGC-3' (forward), and 5'-ACGCGTCGACTTACTTGTACAGCTCGTCCATG-3' (reverse), and then digested with EcoRI and SalI. The two fragments were ligated to pcDNA3.1(+) (Invitrogen, Carlsbad, CA) digested with BamHI and XhoI.

To generate pcSceGFP-300 and pcSceGFP-1.6k, an irrelevant $300-\mathrm{bp}$ or $1.6-\mathrm{kb}$ fragment of the lacZ gene was amplified from pcDNA3.1/His/lacZ (Invitrogen) and ligated into the EcoRI site of pcSceGFP-24.

To generate pcEGFP, the EGFP gene was amplified from pEGFP-C1 using primers: 5'-CGGGATCCGCCACCATGGTGAGCAA-3' (forward), and 5'-ACGCGTCGACTTACTTGTACAGCTCGTCCATG-3' (reverse). The PCR fragment was digested with BamHI and SalI, and was ligated to pcDNA3.1(+) (Invitrogen) digested with BamHI and XhoI.

\section{SSOS}

SSOs were designed to have equal lengths to homologous sequences flanking the insertion fragments in pcSceGFP-24/300/1.6k. SSOs termed with an initiating " $\mathrm{M}$ " indicated the presence of six phosphorothioatemodified nucleotides at each end. SSOs termed with an ending "s" or "as" indicated they were sense or antisense according to the EGFP coding sequence. An irrelevant SSO E6 was used as a control. For complete SSOs sequences, please refer to the Supplementary Data.

\section{Cell lines}

To set up HEK-24/300/1.6k cells or HeLa-24/300/1.6k cells, pcSceGFP-24/300/1.6k plasmids were linearized with $P v u \mathrm{I}$ and transfected into HEK293 or HeLa cells, respectively. The cells were cultured for 48 hours and then passaged at 50,000 cells per 10-cm dish for G418 (500 $\mu \mathrm{g} / \mathrm{mL}$ ) selection.

siRNAs

We transiently transfected the following siRNA duplexes into HEK-24 cells to knock down the targeted genes expression (leading strands): siATM, 5'-AACATACTACTCAAAGACATTdTdT-3' (Andreassen et al., 2004); siATR, 5'-AAGACGGTGTGCTCATGCGGCdTdT-3' (Shiotani et al., 2006); and siDNA-PKcs, 5' AGGGCCAAGCTGTCACTCTdTdT-3' (Feng et al., 2004). The control siRNA was selected as a nontarget sequence: 5'-AGTACGAGAACCTGAAGTAdTdT-3'. All of the siRNA duplexes were synthesized by Dharmacon (Boulder, CO). Cells in 24-well plate were transfected with 40 ng siRNA duplexes by lipofectamine 2000 (Invitrogen). To examine the gene-silencing effect, cells were collected at 24 hours or 48 hours after transfection and subjected to 5\% SDS-PAGE. For immunobloting, anti-ATM, anti-ATR (both are gifts from Dr. Junjie Chen) or anti-DNA-PKcs (Lab Vision, Freemont, CA) was used as the first antibody. To ensure the equal loading, anti- $\gamma$-tubulin (Sigma, St. Louis, MO) was used as an internal control.

\section{Deletion assays}

Cells were seeded to $70 \%$ to $80 \%$ confluence in $24-$ well plates and grown for 24 hours before transfection. For deletion assays on plasmid, SSOs $(0.15 \mu \mathrm{g})$ and pcSceGFPs $(0.75 \mu \mathrm{g})$ with or without I-SceI (New England Biolabs, Beverly, MA) linearization were cotransfected into cells. For deletion assay on genomic DNA, SSOs $(0.15 \mu \mathrm{g})$ with or without I-SceI expression vector $(0.75$ $\mu \mathrm{g}$ ), and with or without siRNAs (40 ng) were transfected into reporter cell lines. For each well, $2 \mu \mathrm{L}$ Lipo-

FIG. 1. Illustration of the SSO-mediated deletion system. EGFP expression was driven by the CMV promoter (arrow). A 24-bp, 300-bp, or 1.6-kb fragment was inserted into +96 locus after start codon ATG within EGFP gene (gray). At the beginning of the insertion, an I-SceI recognition site was introduced, which also introduced two in-frame stop codons. A DSB would be induced at this site upon I-SceI encoding plasmid transfection. SSOs designed to make deletions had equal length of homologous flanking in the inserted fragments. (A) Observation of SSO-mediated deletion on pSceGFP-24 in HeLa cells under a fluorescent microscope. The left panel presents only pSceGFP-24-transfected cells. The right panel presents pSceGFP-24- and SSO M90as-cotransfected cells. (B) The efficiency of the deletion assay can be measured by FACS. After SSO-mediated deletion on pSceGFP-24 in Huh7 cells, the cells were trypsinized and subjected to FACS analysis. The left panel presents only pSceGFP-24-transfected cells. The right panel presents pSceGFP-24- and SSO M90as-cotransfected cells. (C) Observation of SSO-mediated deletion HEK-24 cells under fluorescent microscope. The left panel presents control SSO E6-transfected cells. The right panel presents SSO M90astransfected cells. 


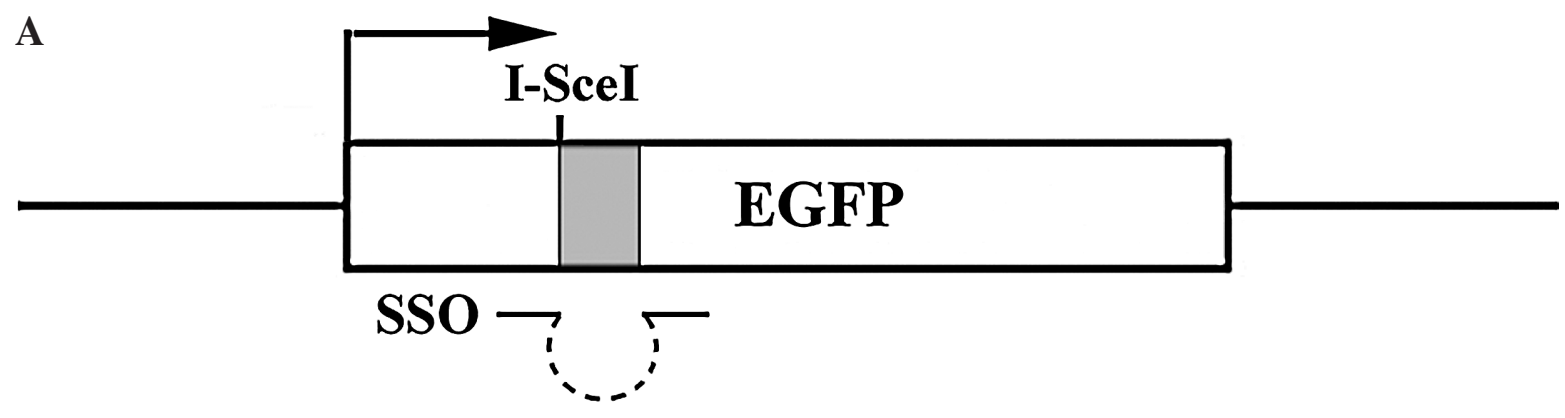

B control

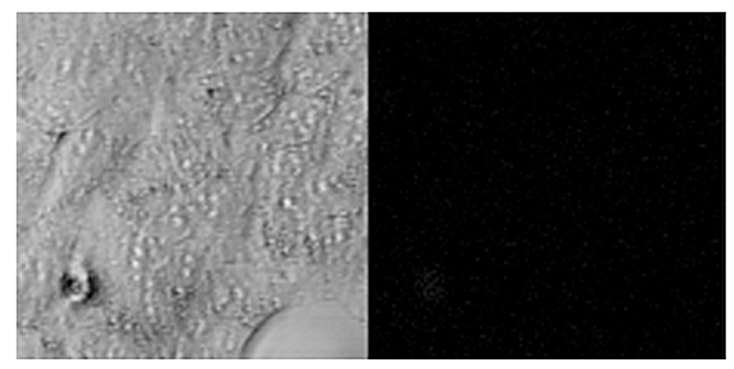

C control

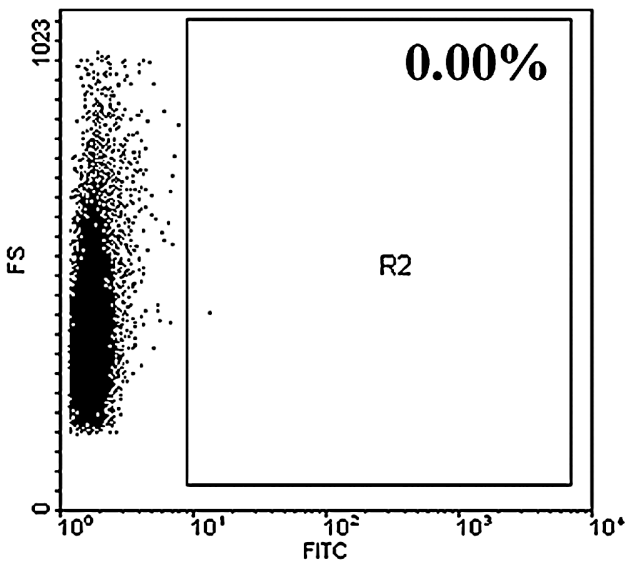

D control

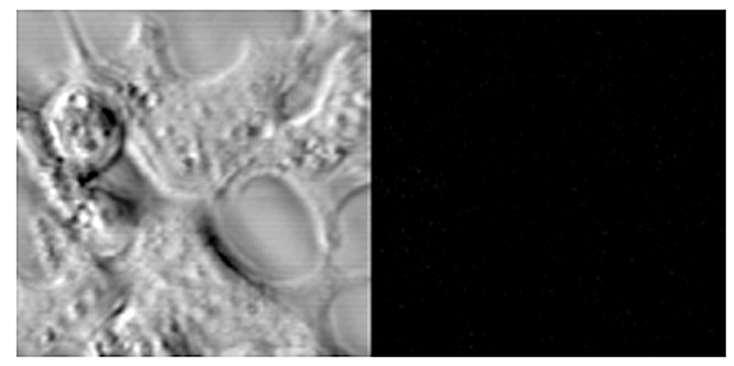

M90as

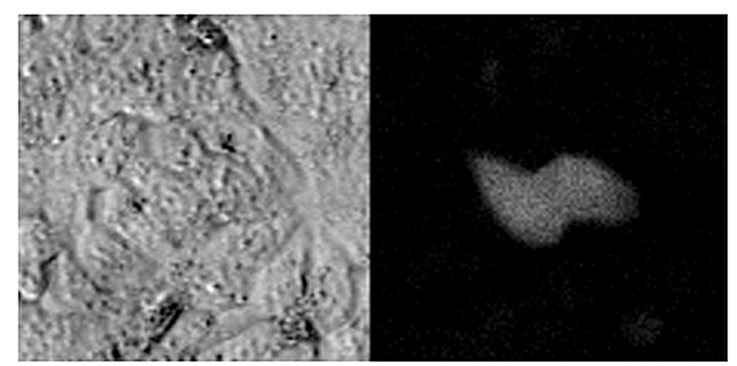

M90as

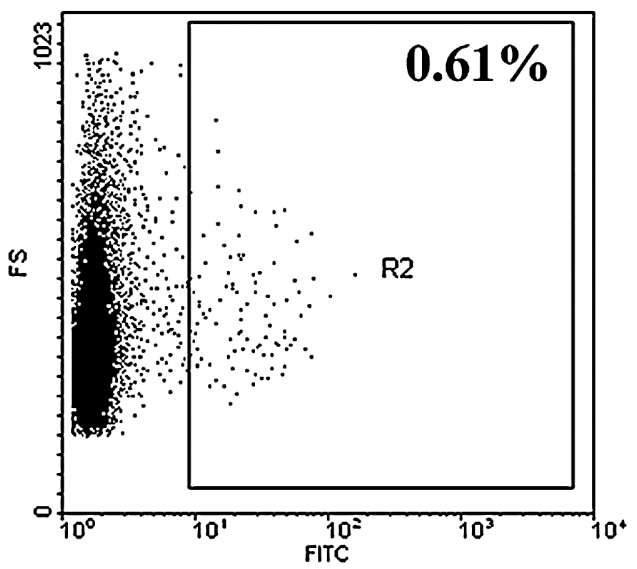

M90as

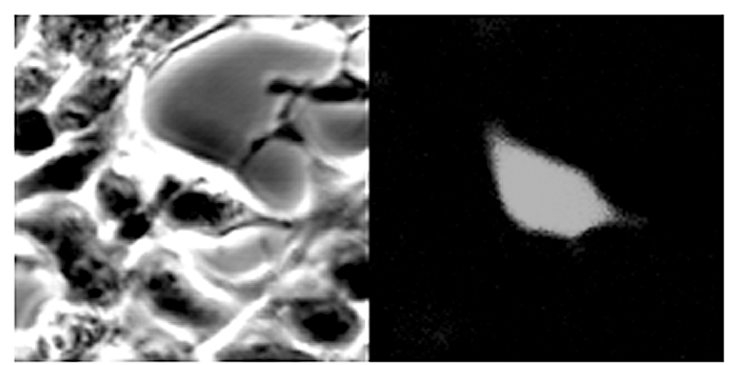

FIG. 1. 
fectamine2000 (Invitrogen) was used according to the protocol. Different drugs, thymidine $(2 \mathrm{mM})$, caffeine (3 $\mathrm{mM})$, pentoxifylline $(3 \mathrm{mM})$, and vanillin $(1 \mathrm{mM})$ (all from Sigma) were added to the culture medium before transfection where indicated.

For deletion assay using double-stranded DNA (dsDNA) as a donor, a PCR fragment amplified from pcEGFP using primers TGGGCGTGGATAGCGGTTT (forward) and CGGGATCCCTTGAAGTTCACCTTGATGC (reverse) was transfected into the cells as a replacement of SSOs.

\section{Microscopy}

Cells after gene repair were detected using an inverted fluorescence microscope (Zeiss Axiovert 135, Thornwood, NY).

\section{FACS analysis}

To measure EGFP repair efficiency, cells were trypsinized 72 hours posttransfection, washed with PBS, and directly subjected to flow cytometry (Beckman Coulter, Fullerton, CA) using a 488-nm argon-ion laser. For each analysis, 20,000 cells were counted. Single cell population was ensured by FS/SS gating during analysis.

\section{RESULTS}

SSO can generate deletions on episomal and genomic DNA without DSB induction

To investigate whether SSOs can generate DNA deletion in mammalian cells, we constructed three EGFP reporters with a 24-bp, 300-bp, or 1.6-kb insertion called pcSceGFP-24/300/1.6k, respectively. At the beginning of the insertion, an I-SceI endonuclease recognition site was included that also introduced two in-frame stop codons to terminate EGFP translation (Fig. 1A). Thus, EGFP expression can only be achieved if the inserted fragment is removed and the EGFP coding sequence was recovered.

We first examined whether SSOs can generate deletions on a plasmid. We transfected pcSceGFP-24 alone or together with SSO M90as into HeLa cells. Seventytwo hours after transfection, cells were observed under a fluorescent microscope. We found that little cells are green in pcSceGFP-transfected cells, while green fluorescence cells can be observed in pcSceGFP-24 and M90as cotransfected cells (Fig. 1B), indicating deletion of the insertion fragment occurred. We also tested HEK293 cells and Huh7 cells and observed deletion events in all of these cell lines. FACS analysis showed the deletion efficiency was about $0.2 \%, 0.1 \%$, and up to
0.6\% in HeLa, HEK293, and Huh7 cells, respectively, while the control group with only pcSceGFP-24 transfection showed $<0.01 \%$ of the cells gave out weak green fluorescence (Fig. 1C). As the transgene expression level was quite high in Huh7 cells as measured by pEGFP-C1 expression (data not shown), the higher deletion efficiency observed in Huh7 cells may be due to a higher read-out efficiency, as FACS analysis is based on fluorescent protein expression level.

Next, we examined whether the SSOs can generate deletions in genomic DNA in mammalian cells. We transfected pcSceGFP-24/300/1.6k into HeLa or HEK293 cells and obtained stable cell lines with chromosomal integration of EGFP reporters after drug selection. Without SSO transfection, or with control SSO E6 transfection, no green cells can be observed in HEK-24 cells, showing a clear background (Fig. 1D). We transfected SSO M90as into HEK-24 cells, and after 72 hours, a small number of green cells were observed under a fluorescent microscope (Fig. 1D), indicating the deletion occurred at the genomic DNA level. However, FACS analysis showed the efficiency was only $<0.01 \%$ (data not shown), and the efficiency was not stable among independent experiments. Despite the low efficiency, we found that the repaired green cells were unable to divide and remained as single cells (Fig. 1D). This phenomenon is similar to that we observed in cells after gene repair by SSO-mediated single nucleotide substitution, in which the repaired cells were arrested in the G2/M phase (Wang et al., 2006).

\section{DNA DSB can be repaired by SSOs and serves as a stimulation signal}

DSBs in human cell genome can be repaired by two major pathways. It is primarily repaired by a nonhomologous end joining (NHEJ) pathway. NHEJ directly ligates two DNA ends that may lead to short deletions, and is therefore potentially mutagenic. DNA-dependent protein kinase (DNA-PK) functions in NHEJ, which comprises the DNA end-binding heterodimer Ku70/Ku80 and the catalytic subunit DNA-PKcs (Jackson, 2002). Instead of NHEJ, human cells may use homologous recombination (HR) to repair DSBs, which utilizes an homologous sequence from an undamaged chromatid, and is thought to be potentially correct. In homologous recombination repair (HRR), ATM/ATR plays a central role, which serve as sensor proteins to passage the DNA damage signal to the downstream effectors (reviewed in Shiloh, 2003; Bartek et al., 2004).

To investigate the role of DSB in SSO-mediated DNA deletion, we transfected the I-SceI expression vector into HEK-24 cells to generate a DSB at its recognition site about 96 bp downstream of the EGFP start codon, and studied the deletion efficiency with SSO transfection. In 
the control experiment, I-SceI expression alone did not lead to functional EGFP expression as measured by FACS analysis or microscopy. It is most likely that DSBs are repaired by the NHEJ pathway without regenerating a functional EGFP gene; our deletion reporter system showed an extremely low background. Then we cotransfected HEK-24 cells with SSOs and I-SceI expression vector. Seventy-two hours later, up to $0.25 \%$ of the cells were repaired as measured by FACS, a $>20$-fold increase than in cells without DSB induction (Table 1).

We also tested whether DSB can stimulate deletion repair on plasmid DNA. We performed the deletion assay on I-SceI linearized or unlinearized plasmids in HEK293 cells. As shown in Table 1, the deletion efficiency was about $0.2 \%$ at the highest for nonlinearized pSceGFP-24. However, for linearized pSceGFP-24, the efficiency was about $10 \%$ in SSO M90as transfected cells. In other comparable groups, plasmid linearization consistently resulted in 10- to 100-fold increases in efficiency. Taken together, these data indicate that DSB can stimulate both genomic and plasmid repair by SSO in mammalian cells.

\section{Cells remained viable after DSB-induced SSO-mediated deletion repair on genomic DNA}

As it is known that cells after DSB-induced HRR can undergo the normal cell cycle (Wang et al., 2006), we investigated the impact of DSB-induced SSO-mediated deletion repair. We performed deletion assays in HEK-24 cells with DSB induction. As a result, we found that the repaired green cells were able to divide and form colonies (Fig. 2A).

We isolated the green cells by fluorescent sorting after expansion, and extracted genomic DNA from the cells for sequencing. We used PCR to amplify the EGFP gene and used a reverse primer for sequencing. As shown in Figure 2B, the artificial insertion was successfully removed, that is, the genomic DNA from the green cells was identical to the EGFP sequence. A weak signal of a second DNA sequence was also observed in the sequenc- ing result, identical to the pcSceGFP-24 sequence. This may be due to heterogeneity in the sorted cells, as the sorted cells were only $80 \%$ fluorescent positive as analyzed by FACS (data not shown).

\section{$D S B$-induced SSO-mediated deletion repair relies on the ATM/ATR pathway}

By using two well-characterized ATM/ATR inhibitors caffeine (Sarkaria et al., 1999) and pentoxifylline (Bohm et al., 2003), we showed that the ATM/ATR pathway strongly regulates DSB-induced HRR in reporter cell lines; however, it is dispensable for SSO-mediated gene repair in the absence of DSB (Wang et al., 2006). Here, we used the same approach to study whether DSB-induced SSO-mediated deletion repair depends on the ATM/ATR pathway as is the case for dsDNA-mediated repair. We used a 722-bp PCR fragment spanning the DSB site as the donor dsDNA. After transfection of this dsDNA fragment into HEK-24 cells, there was no gene repair (fluorescence) without DSB induction, but there were $>0.6 \%$ EGFP-positive cells upon DSB induction. In a separate experiment, M90as was used as SSO donor. The deletion efficiency is about $0.2 \%$. Different inhibitors were added prior to transfection and maintained for 72 hours before flow cytometry analysis.

As shown in Figure 3A and B, two ATM/ATR inhibitors caffeine and pentoxifylline could inhibit both SSOand dsDNA-mediated deletion repairs by about $80 \%$. As a control, vanillin (Durant and Karran, 2003), an inhibitor of DNA-PK in the NHEJ pathway, only caused about $30 \%$ reduction of efficiency in both SSO- and dsDNAmediated deletion repair. All these drugs had little effect on transfection efficiency as inspected by pEGFP-C1 (Clontech) transfection (data not shown). These data indicate that DSB-induced SSO-mediated deletion repair may utilize the ATM/ATR pathway, which is similar to dsDNA-mediated HRR.

To exclude the side effects of these chemicals and further verify the role of ATM/ATR in the repair process,

TABle 1. Deletion EfFiciency

\begin{tabular}{lcllll}
\hline & \multicolumn{4}{c}{ Efficiency $(\%)$} \\
\cline { 2 - 3 } \cline { 5 - 6 } I-SceI & - & & \multicolumn{2}{c}{ HEK-24 + SSOs } \\
\cline { 2 - 3 } \cline { 5 - 6 } M90s & $0.09( \pm 0.01)$ & $12.38( \pm 1.11)$ & & $0.00( \pm 0.00)$ & $0.05( \pm 0.02)$ \\
M90as & $0.20( \pm 0.02)$ & $10.40( \pm 1.24)$ & & $0.01( \pm 0.01)$ & $0.25( \pm 0.03)$ \\
\hline
\end{tabular}

For deletion assay on episomal DNA, HEK293 cells were transfected with SSO and pSceGFP-24 with or without linearization. For deletion assay on genomic DNA, HEK-24 cells were transfected with SSOs alone or cotransfected with I-SceI expression vector. Data were derived from three independent experiments. 

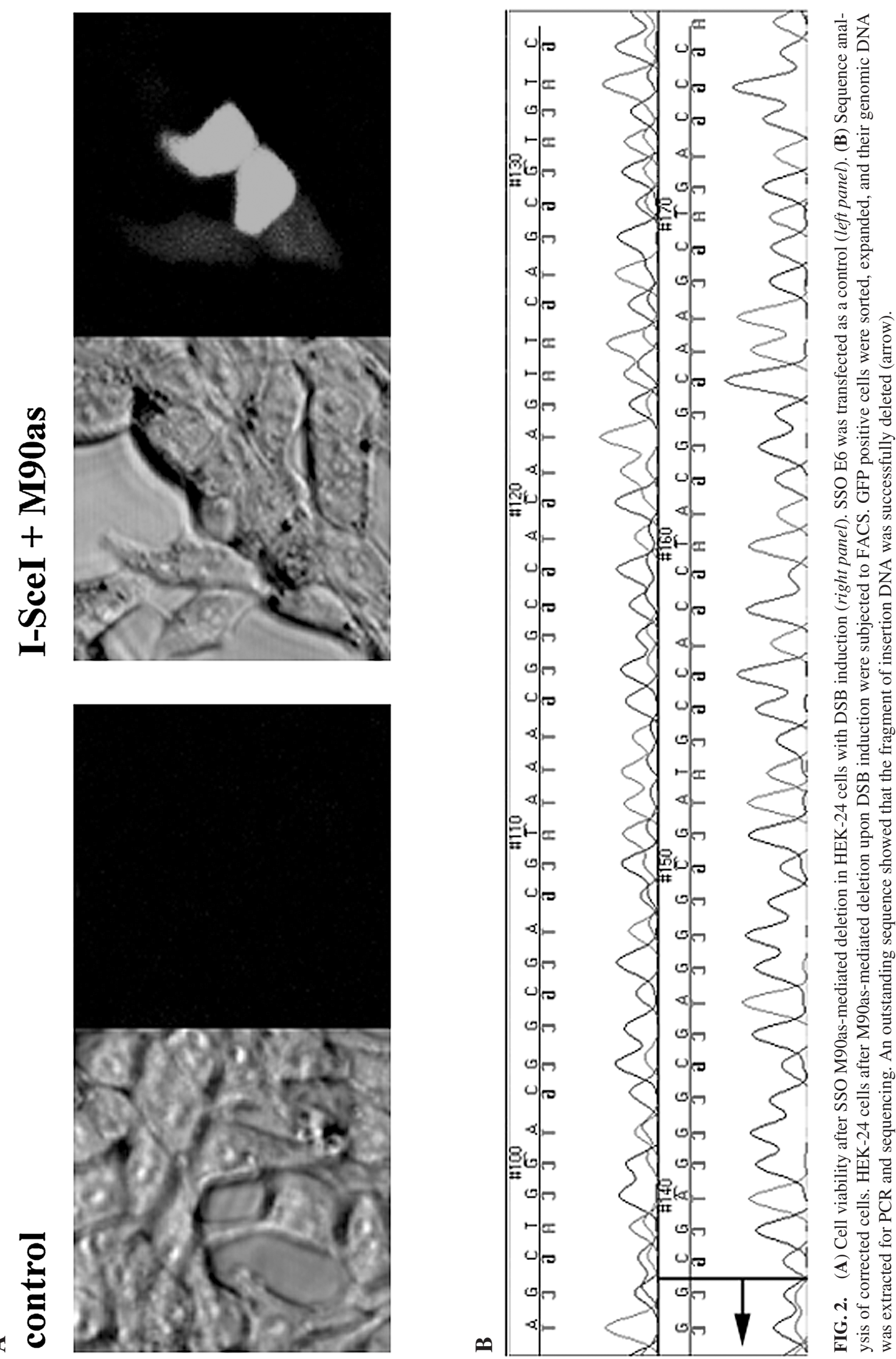
A

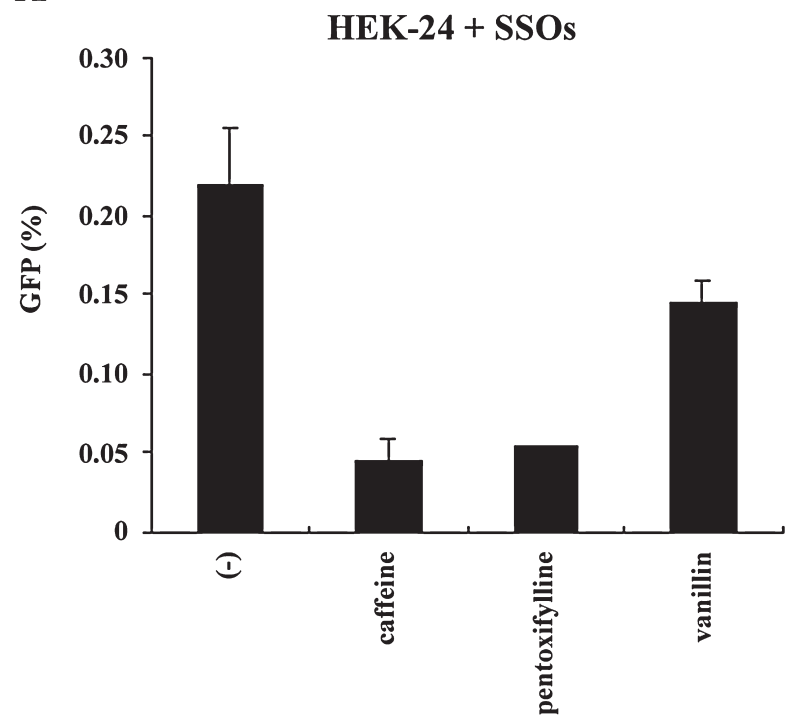

C

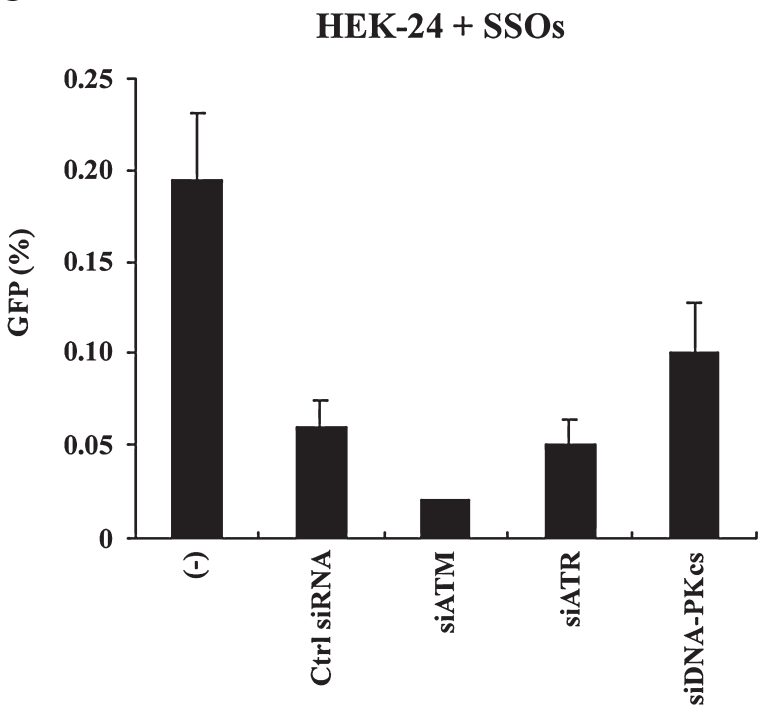

B

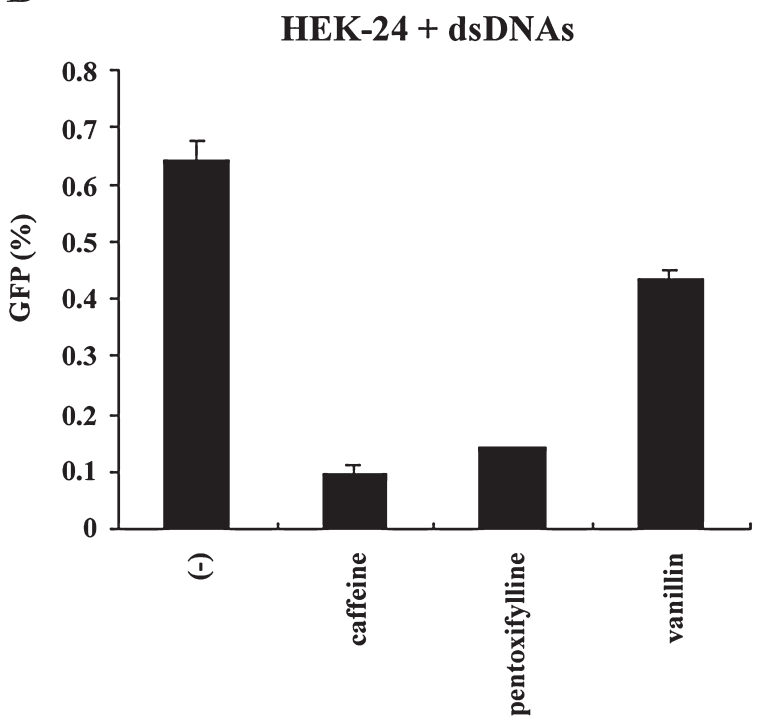

D

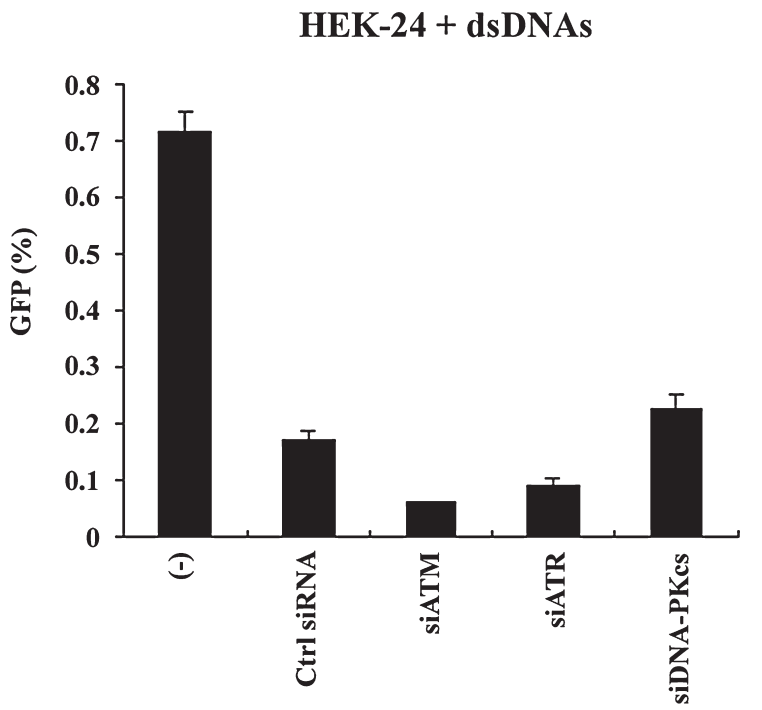

FIG. 3. Deletion in genomic DNA mediated by SSOs or dsDNAs upon DSB induction share similar mechanism. (A,B) HEK-24 cells were treated with the ATM/ATR inhibitors caffeine or pentoxifylline, or with the DNA-PK inhibitor vanillin before cotransfection of I-SceI expression vectors and donor DNA as SSO M90as (A) or dsDNA donors (B). The control experiment (-) indicated that no inhibitor was added before transfection. (C,D) HEK-24 cells were cotransfected with siRNAs and I-SceI expression vectors, together with SSO M90as (C) or dsDNA fragments (D). The control experiment $(-)$ indicated that no siRNA was cotransfected with I-SceI expression vectors and donor DNAs. Data were derived from three independent experiments.

we next used siRNA duplexes, which target ATM, ATR, or DNA-PKcs to silence the corresponding gene expression. Inspected by immonubloting, we found that 24 hours after siRNAs transfection, the targeted gene expression started to decrease (data not shown), and we can observe about $70 \%$ reduction of the protein amount at 48 hours posttransfection (Suppl. Fig. 1). In the deletion assays, we cotransfected siRNAs and I-SceI expression vectors, together with SSO M90as or dsDNA donors. Re- pair efficiency was measured at 72 hours posttransfection. By cotransfecting siRNAs and pEGFP-C1, we found that transfection of siRNAs did not affect the transfection efficiency or the EGFP expression level (data not shown). However, transfection of siRNAs had a nonspecific inhibition effect on repair efficiency, as exhibited by control siRNA (Fig. 3C and D). Compared to the control siRNA, siATM and siATR both inhibited repair efficiency on SSO- and dsDNA-mediated deletion repair. On 
the contrary, the siDNA-PKcs even increased the repair efficiency with both type of donor DNAs (Fig. 3C and D). This result was reasonable because as HR and NHEJ pathways are two major pathways for DSB repair, inhibition of the NHEJ pathway may provide more repair opportunity for HR machinery. Taken together, we verified that ATM/ATR pathway played a central role in DSBinduced deletion repair with donor DNA as SSOs or dsDNAs.

\section{Evaluation of several parameters in SSO-mediated deletion}

In SSO-mediated repair in mammalian cells, several parameters such as SSO modification, SSO length, and strand bias are known to influence the repair efficiency (review in Parekh-Olmedo et al., 2005). Here, we investigate these factors in SSO-mediated deletion, and also investigate the influence of the length of DNA sequence to be deleted.

To test the relationship between deletion efficiency and deletion length, we designed three different lengths of insertion fragment to be deleted by SSOs: $24 \mathrm{bp}, 300$ $\mathrm{bp}$, and $1.6 \mathrm{~kb}$. We observed that on both the plasmid and genome, deletion of the longer fragment proved to be less efficient. On a nonlinearized plasmid, deletion of $24 \mathrm{bp}$ was twofold more efficient than a deletion of $300 \mathrm{bp}$, and about fourfold more efficient than a deletion of $1.6 \mathrm{~kb}$ (Fig. 4A). On linearized plasmids, a similar relationship between deletion efficiency and deletion length was observed (Fig. 4B). For genomic deletion in HeLa24/300/1.6k cells, a low background was observed in HeLa-300/1.6k cells, for which the reason was not clear. SSOs could generate deletion of the 24-bp insertion with the efficiency up to $0.18 \%$. However, for the 300 -bp or $1.6-\mathrm{kb}$ fragments deletion, SSOs can only generate up to $0.01 \%$ repair events compared to the background (Fig. 4C). In HEK-24/300/1.6kb cells with DSB induction, the SSO generated deletion of 24 bp in HEK-24 cells had the highest efficiency. Deletion efficiency for the 300-bp insertion was fivefold lower comparatively, and there was no detectable deletion for the 1.6-kb insertion (Fig. 4D).

To test the effect of different SSO lengths on deletion efficiency, we used 30-, 60-, or 90-mer SSOs in the dele-

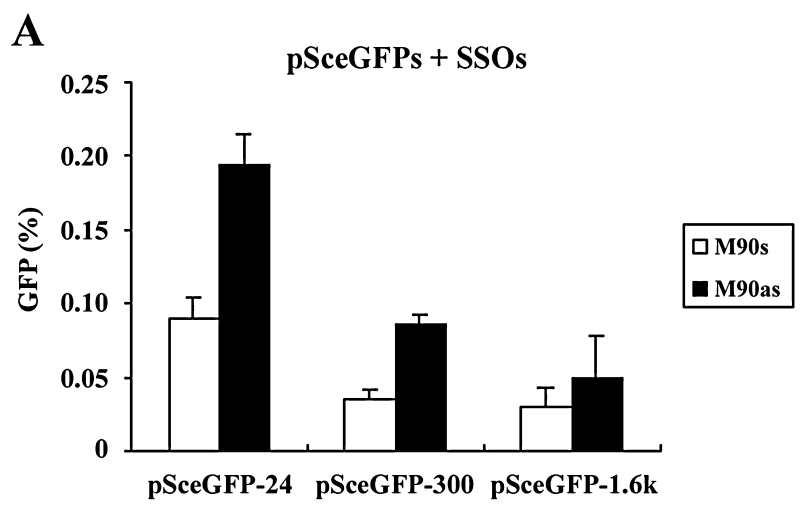

\section{B}
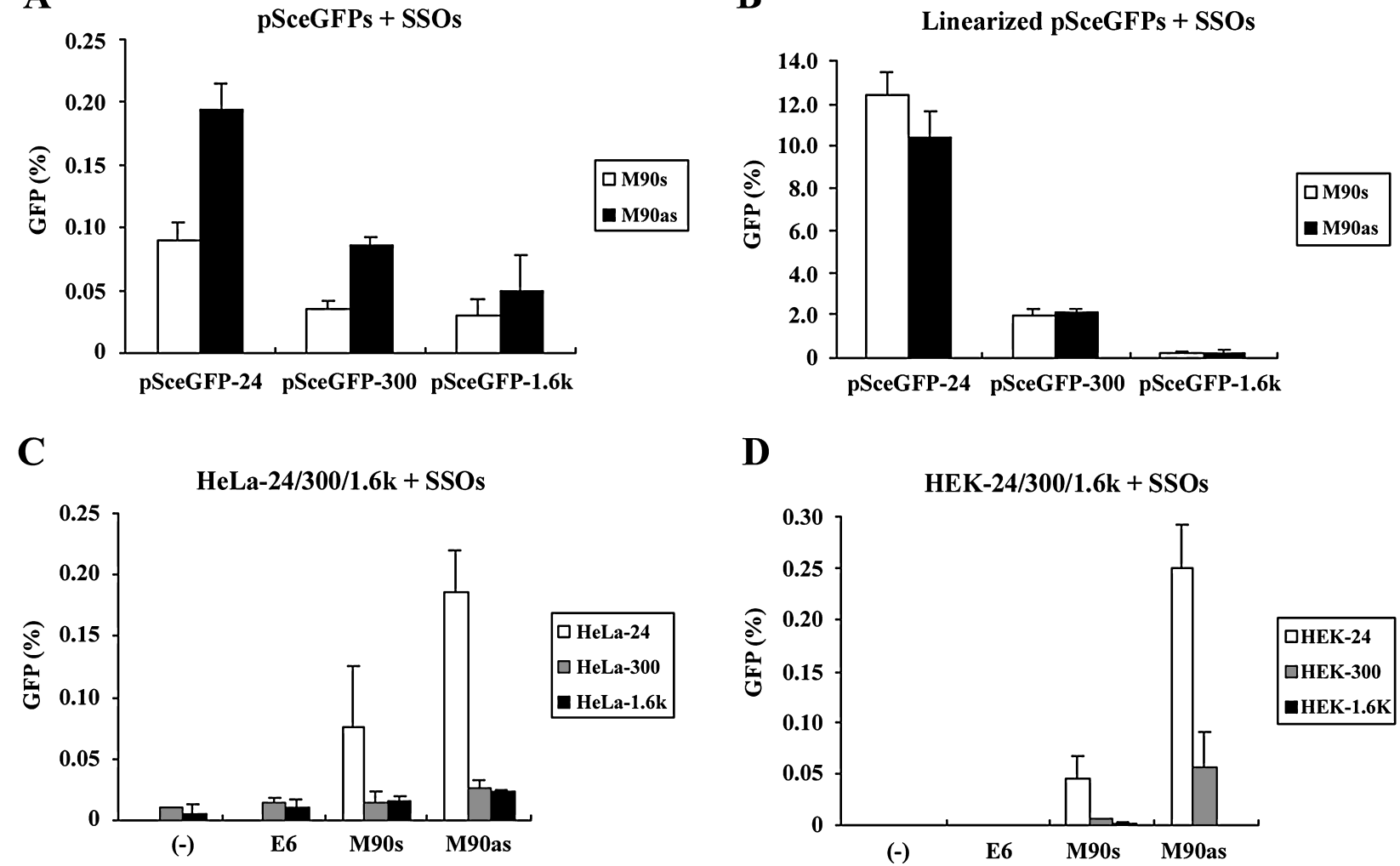

FIG. 4. Effect of deletion length on SSO-mediated deletion efficiency. Factors of sense (empty) or antisense (filled) of SSO were also tested. (A) Deletion of different lengths of the fragment (24 bp, $300 \mathrm{bp}$, or $1.6 \mathrm{~kb}$ ) on unlinearized plasmids in Huh7 cells. (B) Deletion of $24 \mathrm{bp}, 300 \mathrm{bp}$, or $1.6 \mathrm{~kb}$ fragments on linearized plasmids in Huh7 cells. (C) Deletion of different lengths of fragment in HeLa-24 (white), HeLa-300 (gray), or HeLa-1.6k (black) cells with DSB induction. (D) Deletion of different lengths of fragments in HEK-24 (white), HEK-300 (gray), or HEK-1.6k (black) cells with DSB induction. Control experiments included no SSO transfected cells (-), or unrelated SSO E6-transfected cells. Data were derived from three independent experiments. 

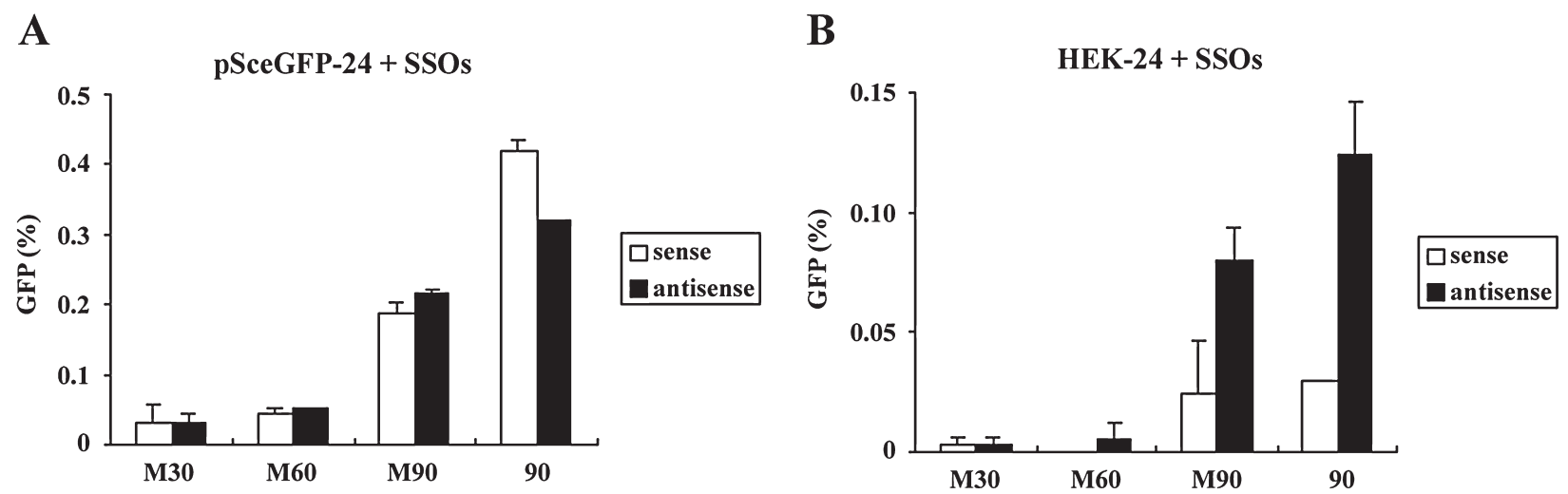

FIG. 5. Effect of SSO length on deletion efficiency. (A) pSceGFP-24 and SSOs were cotransfected into HEK293 cells. (B) HEK-24 cells were cotransfected with SSOs and I-SceI expression vector. Factors of sense (white) or antisense (black) of SSOs were also tested. Data were derived from three independent experiments.

tion assay. Longer SSOs always showed a higher efficiency at both the episome and genome level. 90-mer SSO was the most efficient for the SSO tested, followed by 60 -mer SSO, and finally the 30-mer SSO, which showed the lowest efficiency (Fig. 5A and B). This clearly indicates that the deletion efficiency is positively related to the length of homologous sequence.

To test whether there is strand bias in SSO-mediated deletions, we used sense or antisense SSO to make deletions on plasmids or genomic DNA. Previously we showed that only antisense SSOs can make single nucleotide substitutions in genomic DNA, indicating a strong strand bias (Yin et al., 2005). In contrast, we found here that both sense and antisense SSOs can generate deletion on plasmid and genomic DNA (Figs. 4 and 5). For plasmids, there was no obvious difference in deletion efficiency between sense and antisense SSO treatment (Fig. $4 \mathrm{~A}$ and $5 \mathrm{~A})$. However, when targeting genomic DNA, antisense SSOs always showed a higher efficiency than their sense counterparts (Fig. 4B and 5B).

A

pSceGFP-24 + SSOs

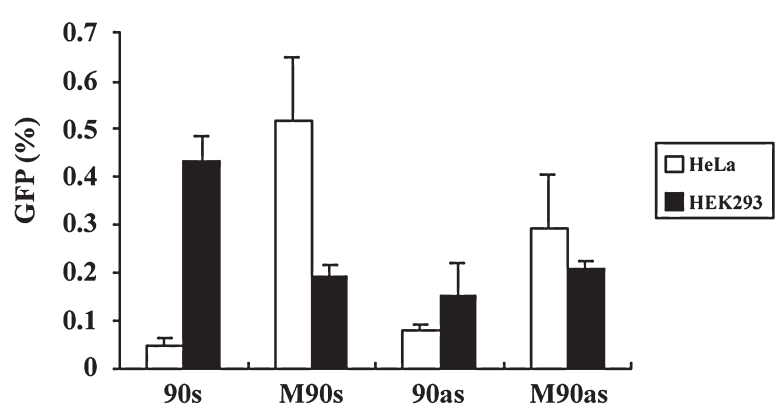

We also tested whether phosphorothioate modification can increase SSO-mediated deletion efficiency by protecting SSOs from DNA nuclease. Surprisingly, we found that although efficiency was similar in HEK293 cells, in HeLa cells, modified SSOs exhibited a much higher efficiency than unmodified ones (Fig. 6A and B). This result was consistent in both plasmid and genome based assays. As phosphorothioate modification functions to protect SSOs against nucleases, we suggest that the nuclease activity may vary among cell types, and the effect of SSO modification might be determined by different DNA nuclease activity in different cell types.

\section{DISCUSSION}

In this study we successfully generated up to $1.6-\mathrm{kb}$ DNA fragment deletions in mammalian cells using SSOs. We demonstrated that transfection of SSOs with flanking homologous sequences could target homolo-

B

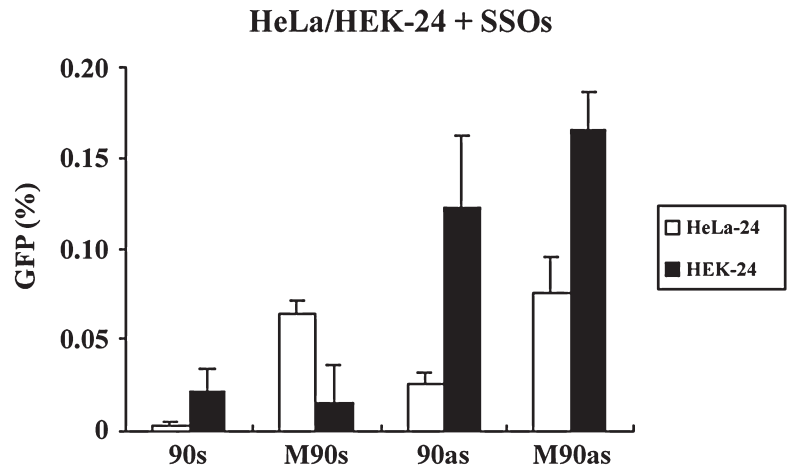

FIG. 6. Effect of SSO modification on deletion efficiency was influenced by cell type. SSOs with or without modification were used to perform deletion assays on pSceGFP-24 in HeLa (empty) or HEK293 (filled) cells (A) or in HeLa-24 (white) or HEK-24 (black) cells with DSB induction $(\mathbf{B})$. Data were derived from three independent experiments. 
gous loci and cause deletions in both plasmid and genomic DNA. The deletion efficiency was about $0.2 \%$ for plasmids, and up to $0.01 \%$ for chromosomal DNA.

In bacteria, SSOs can generate deletions very efficiently with the aid of a $\lambda$-phage-encoded beta protein, and the genetically modified bacteria can go through the cell cycle and form colonies (unpublished data). However, in mammalian cells, although SSO-mediated deletion can be achieved, the deletion efficiency is relatively low. Furthermore, cells after chromosomal DNA deletion cannot divide to form colonies, which is similar to cell fate after SSO-mediated single-base substitution as previously reported (Olsen et al., 2005a; Wang et al., 2006). We demonstrate here that this "survival" obstacle can be overcome by introducing a DSB at the target site, which results in viable cells following genomic DNA deletion by SSOs. These results indicate that SSO-mediated deletion uses a different mechanism in mammalian cells to the bacterial recombineering system.

DSB also significantly increased SSO-mediated deletion efficiency, and we demonstrated that this process depends on the ATM/ATR kinases, similarly to DSB-induced dsDNA-mediated repair. To our knowledge, in the DSB-induced HRR process, the DNA terminates at the original break-point are first digested by the DNA exonuclease, leaving single-stranded regions. Repair proteins recruited to DSB can bind to these single-stranded ends and facilitate pairing and strand exchange with their homologous counterparts. According to the current model, the two single-stranded ends both pair to their homologous partners, synthesize new DNA sequence, separate from the complex and fill the gap to complete the HRR process (reviewed in Bartek et al., 2004). Here, we found DSB can also be repaired using only SSO, suggesting that repair of one end may be sufficient for the HRR process. We prefer the model proposed by Storici et al. (2006) that the SSO pairs to one single-strand overhang at the break site and serves as a replication template. The paired DNA overhang synthesizes new nucleotides, which can pair to another overhang at the break site, leading to completion of the HRR process.

In this report, we identified several factors that affect the efficiency of SSO-mediated deletion repair. The efficiency proved to be negatively related to deletion length, but positively related to SSO length. This result of SSO length was expected, as in the HRR system increasing double-stranded donor DNA length results in a higher recombination efficiency (Bibikova et al., 2003). This is in contrast to the SSO-mediated point mutation system without DSB induction, in which it was always the short SSOs around 25- to 45-mer long achieving the highest efficiency (Igoucheva et al., 2001; Kenner et al., 2004; Olsen et al., 2005a, 2005b; Yin et al., 2005), implying different mechanisms were used.

Strand bias is another factor previously identified to influence repair efficiency, as antisense SSOs always show much higher efficiency than sense SSOs in repair assays (Igoucheva et al., 2001, 2003; Yin et al., 2005). Here we showed both sense and antisense SSO were able to achieve successful deletion repair on plasmids with or without DSB induction, and in genomic DNA with DSB induction. There was no strand bias on plasmid repair, but on genomic DNA, antisense SSOs always showed a higher efficiency than sense SSOs.

We found SSO modification also influences repair efficiency, and this is related to cell type. In HeLa but not HEK293 cells, modified SSOs showed significantly higher efficiency than unmodified ones. As phosphorothioate modifications protect SSO against nuclease degradation, we suggest the nuclease activity varies among cell types. Modified SSOs have always showed much higher repair ability in previous reports (Igoucheva et al., 2001; Yin et al., 2005). However, modified and unmodified SSOs were reported to have similar efficiency for mutagenesis in HEK293 cells (Radecke et al., 2004). More research using various cell types is needed to confirm our hypothesis.

Concerning cell viability after chromosomal repair, we found that cells can divide to form colonies after DSB-induced repair by SSO, which implies that future widespread application of this approach may be feasible. As zinc-finger nuclease (ZFN) was reported to be a powerful tool to generate DSB at prechosen sites in the genome (Bibikova et al., 2003), we suggest that it may be possible to combine the SSO approach with ZFN to allow easier design and engineering of genomic DNA in mammalian cells.

\section{ACKNOWLEDGMENTS}

We thank Dr. Maria Jasin for the DR-GFP plasmid and I-SceI expression vector. We thank Dr. Junjie Chen for antibodies against ATM and ATR. We thank Dr. Julian A. Tanner for critical reading the manuscript. This work was supported by a joint grant from the Research Grant Council of Hong Kong and the National Science Foundation to J.D.H. and D.L., and by National Basic Research Program of China Grant 2004CB518803 to D.L.

\section{REFERENCES}

ANDREASSEN, P.R., D'ANDREA, A.D., and TANIGUCHI, T. (2004). ATR couples FANCD2 monoubiquitination to the DNA-damage response. Genes Dev. 18, 1958-1963.

BARTEK, J., LUKAS, C., and LUKAS, J. (2004). Checking on DNA damage in S phase. Nat. Rev. Mol. Cell Biol. 5, 792-804.

BIBIKOVA, M., BEUMER, K., TRAUTMAN, J.K., and CARROLL, D. (2003). Enhancing gene targeting with designed zinc finger nucleases. Science 300, 764. 
BOHM, L., ROOS, W.P., and SERAFIN, A.M. (2003). Inhibition of DNA repair by Pentoxifylline and related methylxanthine derivatives. Toxicology 193, 153-160.

DURANT, S., and KARRAN, P. (2003). Vanillins-a novel family of DNA-PK inhibitors. Nucleic Acids Res. 31, 5501-5512.

FENG, J., PARK, J., CRON, P., HESS, D., and HEMMINGS, B.A. (2004). Identification of a PKB/Akt hydrophobic motif Ser-473 kinase as DNA-dependent protein kinase. J. Biol. Chem. 279, 41189-41196.

IGOUCHEVA, O., ALEXEEV, V., PYRCE, M., and YOON, K. (2001). Targeted gene correction by small single-stranded oligonucleotides in mammalian cells. Gene Ther. 8, 391-399.

IGOUCHEVA, O., ALEXEEV, V., and YOON, K. (2003). Transcription affects formation and processing of intermediates in oligonucleotide-mediated gene alteration. Nucleic Acids Res. 31, 2659-2670.

JACKSON, S.P. (2002). Sensing and repairing DNA doublestrand breaks. Carcinogenesis 23, 687-896.

KENNER, O., LUTOMSKA, A., SPEIT, G., VOGEL, W., and KAUFMANN, D. (2004). Concurrent targeted exchange of three bases in mammalian hprt by oligonucleotides. Biochem. Biophys. Res. Commun. 321, 1017-1023.

LIU, L., RICE, M.C., and KMIEC, E.B. (2001). In vivo gene repair of point and frameshift mutations directed by chimeric RNA/DNA oligonucleotides and modified single-stranded oligonucleotides. Nucleic Acids Res. 29, 4238-4250.

LU, I.L., LIN, C.Y., LIN, S.B., CHEN, S.T., YEH, L.Y., YANG, F.Y., and AU, L.C. (2003). Correction/mutation of acid alpha-D-glucosidase gene by modified single-stranded oligonucleotides: in vitro and in vivo studies. Gene Ther. 10, 1910-1916.

OLSEN, P. A., RANDOL, M., and KRAUSS, S. (2005a). Implications of cell cycle progression on functional sequence correction by short single-stranded DNA oligonucleotides. Gene Ther. 12, 546-551.

OLSEN, P.A., RANDOL, M., LUNA, L., BROWN, T., and KRAUSS, S. (2005b). Genomic sequence correction by single-stranded DNA oligonucleotides: role of DNA synthesis and chemical modifications of the oligonucleotide ends. J. Gene Med. 7, 1534-1544.

PAREKH-OLMEDO, H., FERRARA, L., BRACHMAN, E., and KMIEC, E.B. (2005). Gene therapy progress and prospects: targeted gene repair. Gene Ther. 12, 639-646.

PIERCE, A.J., JOHNSON, R.D., THOMPSON, L.H., and JASIN, M. (1999). XRCC3 promotes homology-directed repair of DNA damage in mammalian cells. Genes Dev. 13, 2633-2638.

RADECKE, F., RADECKE, S., and SCHAWARZ, K. (2004). Unmodified oligodeoxynucleotides require single-strandedness to induce targeted repair of a chromosomal EGFP gene. J. Gene Med. 6, 1257-1271.
RADECKE, F., PETER, I., RADECKE, S., GELLHAUS, K., SCHWARZ, K., and CATHOMEN, T. (2006). Targeted chromosomal gene modification in human cells by singlestranded oligodeoxynucleotides in the presence of a DNA double-strand break. Mol. Ther. 14, 798-808.

SARKARIA, J.N., BUSBY, E.C., TIBBETTS, R.S., ROOS, P., TAYA, Y., KARNITZ, L.M., and ABRAHAM, R.T. (1999). Inhibition of ATM and ATR kinase activities by the radiosensitizing agent, caffeine. Cancer Res. 59, 4375-4382.

SHILOH, Y. (2003). ATM and related protein kinases: safeguarding genome integrity. Nat Rev Cancer 3, 155-168.

SHIOTANI, B., KOBAYASHI, M., WATANABE, M., YAMAMOTO, K., SUGIMURA, T., and WAKABAYASHI, K. (2006). Involvement of the ATR- and ATM-dependent checkpoint responses in cell cycle arrest evoked by pierisin1. Mol. Cancer Res. 4, 125-133.

STORICI, F., DURHAM, C.L., GORDENIN, D.A., and RESNICK, M.A. (2003). Chromosomal site-specific doublestrand breaks are efficiently targeted for repair by oligonucleotides in yeast. Proc Natl Acad Sci USA 100, 14994-14999.

STORICI, F., SNIPE, J.R., CHAN, G.K., GORDENIN, D.A., and RESNICK, M.A. (2006). Conservative repair of a chromosomal double-strand break by single-strand DNA through two steps of annealing. Mol. Cell Biol. 26, 7645-7657.

WANG, Z., ZHOU, Z.J., LIU, D.P., and HUANG, J.D. (2006). Single-stranded oligonucleotide-mediated gene repair in mammalian cells has a mechanism distinct from homologous recombination repair. Biochem. Biophys. Res. Commun. 350, 568-573.

WU, X. S., XIN, L., YIN, W.X., SHANG, X.Y., LU, L., WATT, R.M., CHEAH, K.S., and HUANG, J.D. (2005). Increased efficiency of oligonucleotide-mediated gene repair through slowing replication fork progression. Proc. Natl. Acad. Sci. USA 102, 2508-2513.

YIN, W.X., WU, X.S., LIU, G., LI, Z.H., WATT, R.M., HUANG, J.D., LIU, D.P., and LIANG, C.C. (2005). Targeted correction of a chromosomal point mutation by modified single-stranded oligonucleotides in a GFP recovery system. Biochem. Biophys. Res. Commun. 334, 1032-1041.

Address reprint requests to: Dr. Jian-Dong Huang

Department of Biochemistry 3/F Laboratory Block Faculty of Medicine Building The University of Hong Kong

21 Sassoon Road, Pokfulam

Hong Kong SAR, People's Republic of China

E-mail: jdhuang@hkucc.hku.hk 


\section{SUPPLEMENTAL DATA}

\section{SSO sequences:}

Underlined nucleotides are with phosphorothioate modification.

M30as:

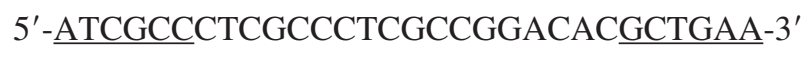

M60as:

5'-CTTGCCGTAGGTGGCATCGCCCTCGCCCTCGCCGGACACGCTGAACTTGTGGCCGTTTAC-3'

M90as:

5'-GAACTTCAGGGTCAGCTTGCCGTAGGTGGCATCGCCCTCGCCCTCGCCGGACACGCTGAACTTGTGGCCGTTTACGTCGCCGTCCAGCTC-3'

90as:

5'-GAACTTCAGGGTCAGCTTGCCGTAGGTGGCATCGCCCTCGCCCTCGCCGGACACGCTGAACTTGTGGCCGTTTACGTCGCCGTCCAGCTC-3'

M30s:

5'-TTCAGCGTGTCCGGCGAGGGCGAGGGCGAT-3'

M60s:

5'-GTAAACGGCCACAAGTTCAGCGTGTCCGGCGAGGGCGAGGGCGATGCCACCTACGGCAAG-3'

M90s:

5'-GAGCTGGACGGCGACGTAAACGGCCACAAGTTCAGCGTGTCCGGCGAGGGCGAGGGCGATGCCACCTACGGCAAGCTGACCCTGAAGTTC-3'

90s:

5'-GAGCTGGACGGCGACGTAAACGGCCACAAGTTCAGCGTGTCCGGCGAGGGCGAGGGCGATGCCACCTACGGCAAGCTGACCCTGAAGTTC-3'

Control SSO:

E6:

5'-CCTTGCTCACCATGGTGGCGGAATT-3'

$\mathbf{A}$

B

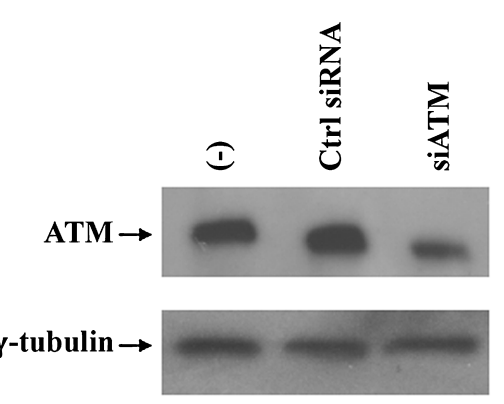

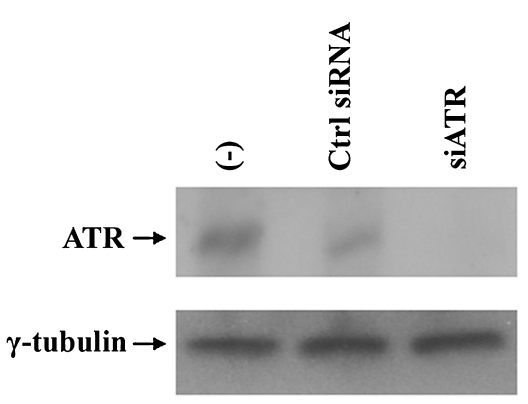

C

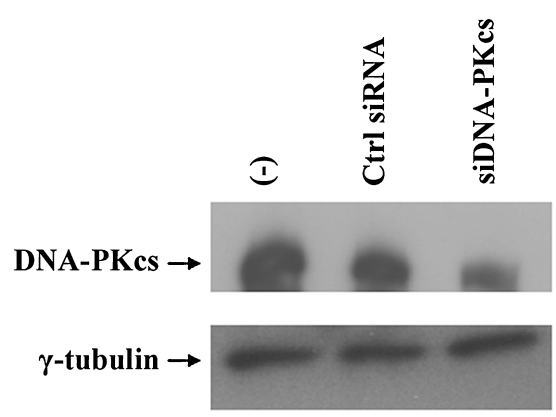

FIG. S1. siRNAs efficiently silenced protein expression. Control siRNA, siATM, siATR, or siDNA-PKcs were transfected into HEK-24 cells separately. Control experiment (-) indicated cells without transfection. Cells were collected at 48 hours after transfection. Immunobloting was performed to examine the targeted protein amount by using corresponding first antibodies: anti-ATM (A), anti-ATR (B), and anti-DNA-PKcs $(\mathbf{C})$, respectively. Anti- $\gamma$-tubulin was used as an internal control to ensure the equal loading. 